\title{
Multiresolution approach based on projection matrices
}

\author{
Javier Vargas* and Juan Antonio Quiroga \\ Optics Department, Universidad Complutense de Madrid, Facultad de CC, Físicas, \\ Ciudad Universitaria s/n, 28040 Madrid, Spain \\ *Corresponding author: jvargas@fis.ucm.es
}

Received 22 September 2008; revised 9 January 2009; accepted 9 January 2009; posted 26 January 2009 (Doc. ID 101853); published 23 February 2009

\begin{abstract}
Active triangulation measurement systems with a rigid geometric configuration are inappropriate for scanning large objects with low measuring tolerances. The reason is that the ratio between the depth recovery error and the lateral extension is a constant that depends on the geometric setup. As a consequence, measuring large areas with low depth recovery error requires the use of multiresolution techniques. We propose a multiresolution technique based on a camera-projector system previously calibrated. The method consists of changing the camera or projector's parameters in order to increase the system depth sensitivity. A subpixel retroprojection error in the self-calibration process and a decrease of approximately one order of magnitude in the depth recovery error can be achieved using the proposed method. (C) 2009 Optical Society of America
\end{abstract}

OCIS codes: $\quad 000.3119,110.0110,120.0120,120.4640,150.0150$.

\section{Introduction}

The knowledge of the three-dimensional (3D) shape of an object is important for a wide range of industrial, technical, and medical applications. Structured light active triangulation measuring systems are well suited for this purpose, because they are fast, noncontact, and whole field. In these techniques a pattern (or a set of patterns) is projected over the object to solve the correspondence problem between the camera and projector image planes. Finally, by means of a previous calibration process, these correspondences are transformed into Cartesian coordinates by a triangulation process [1]. One characteristic of these systems is that the ratio between the depth recovery error and the lateral extension is a constant that depends on the geometric setup. Therefore there is a compromise between the scene lateral size and the depth recovery error. Standard active triangulation techniques are insufficient for simultaneously measuring the global shape and the fine details of an extended object, which is an important point in the quality control of aeronautical or automobile industries. In these applications there has

0003-6935/09/071295-08 $\$ 15.00 / 0$

(C) 2009 Optical Society of America to be an exhaustive control of the shape of extended surfaces as well as of their microstructure; this guarantees the adjustment of the different parts and ensures that there are no defects at the surface. The problem of simultaneously obtaining global and local surface information using structured light techniques can be solved using multiresolution techniques [2-9].

Most of the existing multiresolution methods [2-4] divide the surface into overlapping areas of smaller size, where a local measure, with a low depth recovery error, is performed. Each local measurement must be chosen with a sufficient overlap with its neighbors. For the registration of the different views, one can use markers on the object surface [10] or measure the position of the local sensors [2]. This multiresolution approach has two important problems. First the markers have to be applied onto the surface of the object. Second there might be errors in the registration process, especially in the case of smooth surfaces. An alternative approach consists of a system combining several measurement techniques. Each measuring system is able to achieve profile data in different scales of lateral extensions and depth resolutions [5-8]. In [5-8] a measurement of the whole surface is obtaine $\bar{d} \overline{b y}$ a fringe projection 
system. For the measurement of the low details in the surface, local measurements with a confocal microscope are performed. The automatic registration of the 3D data sets is done because the transformation between the different coordinates systems is well-known by means of an external positioning system. A final refinement process is carried out using the iterative closest point algorithm.

Another multiresolution possibility can be the use of an adaptable fringe projection system. In this case the measuring device is capable of changing its internal and/or external parameters. The main problem of such a system is that it has to be capable of dynamically recalibrating or self-calibrating during the measuring process. Much work has been done to solve the calibration problem for an adaptive system $[9,11-13]$. Note that, in active triangulation methods, the terms "self-calibration" or "recalibration" are used without distinction. In [9] it is not necessary to recalibrate the system explicitly, but this approach has the limitation that it can only be used when the object to scan is piecewise planar. In [11] a selfcalibration approach is used to obtain a $360^{\circ}$ view of the measured object. In this case the self-calibrating approach is based on the overlapping of the phase values between views, making possible the calculation of different orientations (extrinsic parameters) between projectors. The final full-body 3D measurement is done using the previously calibrated (internal parameters) cameras and projectors. In $[12,13]$ a method is presented to dynamically recalibrate the $3 \mathrm{D}$ rig when the relative pose between the camera and the projector is changed. In this case the internal and distortion parameters of the camera and the projector are not changed during the measurement process. For the estimation of the extrinsic parameters, geometric and focus cues are used, and a plane-movement restriction of the camera is imposed. These cues reduce the degree of freedom of the extrinsic parameters but are difficult to measure.

Here we present a multiresolution method based on the computation of the camera or projector projection matrix for an adaptable fringe projection system. The proposed method does not need to divide the image in patches or use any cue. Starting from a calibrated configuration, the $3 \mathrm{D}$ rig can be recalibrated as many times as needed while the camera or the projector changes its relative poses and its internal parameters.

This work is organized in three parts. In Section 2 we present the theoretical foundations. In Section 3 experimental results are shown. Finally in Section $\underline{4}$ conclusions are drawn.

\section{Theoretical Foundations}

\section{A. Three-Dimensional Measurement with a Structured} Light System

An important previous step in any active triangulation measuring process is the calibration procedure of the fringe projection system. The calibration ap- proach involves obtaining the camera and projector calibration parameters that relate a $3 \mathrm{D}$ point with its image in the camera and projector retinal planes. Following [14] the image formation process of a 3D point in the camera retinal plane can be modeled as

$$
s m_{C}=P_{C} M_{W},
$$

where $M_{W}=\left[X_{W}, \mathrm{Y}_{W}, \mathrm{Z}_{W}, 1\right]^{T}$ are the $3 \mathrm{D}$ point homogeneous coordinates, referred in an arbitrary world reference system, $P_{C}$ is the $4 \times 3$ camera projection matrix, $m_{C}=\left[u_{C}, v_{C}, 1\right]^{T}$ are the camera homogeneous coordinates of $M_{W}$, and $s$ is an arbitrary scale factor. An equivalent relation is obtained for the projection of $M_{W}$ in the projector reference system:

$$
s m_{P}=P_{P} M_{W},
$$

where $P_{P}$ is the projector projection matrix, and $m_{P}=\left[u_{P}, v_{P}, 1\right]^{T}$.

The projection matrix is composed of the internal and external parameters [14]. Expanding Eqs. (1) and (2) we obtain

$$
\begin{aligned}
& s m_{C}=K_{C}\left[R_{C} \mid \mathbf{t}_{C}\right] M_{W}, \\
& s m_{P}=K_{P}\left[R_{P} \mid \mathbf{t}_{P}\right] M_{W},
\end{aligned}
$$

where $K_{C}$ and $K_{P}$ are the camera and projector internal matrices, and $\left[R_{C} \mid \mathbf{t}_{C}\right]$ and $\left[R_{P} \mid \mathbf{t}_{P}\right]$ are the camera and projector external parameters, composed of a rotation matrix and a $3 \times 1$ translation vector, that relate the world and camera or projector reference systems.

The internal matrix is an upper triangular matrix and, for the camera and projector, is given by

$$
K_{i}=\left(\begin{array}{ccc}
f_{i}^{X} & 0 & C_{i}^{X} \\
0 & f_{i}^{Y} & C_{i}^{Y} \\
0 & 0 & 1
\end{array}\right),
$$

with $i$ equal to $C$ or $P$ (camera or projector), $f_{i}^{X}$ and $f_{i}^{Y}$ are the focal lengths in terms of pixel dimension, and $C_{i}^{X}$ and $C_{i}^{Y}$ are the principal points coordinates (point where the optical axis of the system meets the image plane). We have considered in Eq. (5) that the skew is equal to zero, as is typically the case.

If we expand the projection matrix, we obtain

$$
P_{i}=\left[K_{i} R_{i} \mid K_{i} \mathbf{t}_{i}\right]
$$

where again $i$ is equal to $C$ or $P$. Note that the three first columns in Eq. (6) correspond to the multiplication of an upper triangular matrix $K_{i}$, with an orthogonal matrix $R_{i}$ that can be split by an $R Q$ factorization [14]. Finally, using the computed parameters of $K_{i}$ and the fourth column of $P_{i}$, the translation vector $\mathbf{t}_{i}$ can be computed. The camera and projector model that we have presented is based on a central projection model. Often a central projection is insufficient to accurately model the camera 
and projector, and it is necessary to model the lens distortion. Typically the most important deviations are radial and tangential distortion that can be modeled as a powers series with two coefficients for each case [14]. In this general case, the calibration process of an active triangulation system consists of obtain$\operatorname{ing} f_{i}^{X}, f_{i}^{Y}, C_{i}^{X}, C_{i}^{Y}, R_{i}, \mathbf{t}_{i}$ for $i$ equal to $C$ or $P$, and the distortion parameters for camera and projector.

Once the "fringe projection system" has been calibrated, we can make a 3D measurement from the correspondences between camera and projector retinal planes. In our case we projected two absolute phases, parallel to the projector $X$ and $Y$ axes, and capture the images. The absolute phases are computed using a combination of gray-code (GC) and phase-shifting (PS) techniques [1-5]. If $\Phi_{X}\left(m_{C}\right)$ and $\Phi_{Y}\left(m_{C}\right)$ are the measured absolute phases at the image point $m_{C}$, the projector coordinates are given by

$$
\left(u_{P}, v_{P}\right)=\left(\frac{\Phi_{X} T_{X}}{2 \pi}, \frac{\Phi_{Y} T_{Y}}{2 \pi}\right)
$$

where $T_{X}$ and $T_{Y}$ are the width in pixels of the projected fringe in the PS method in the retinal projector plane and parallel to the $X$ and $Y$ projector axes.

Since $s u_{C}=P_{C}^{1} M_{W}$ and $s=P_{C}^{3} M_{W}$, with $P_{C}^{n}$ as the $n$th row of $P_{C}$, we can remove the homogeneous scale $s$ in the camera and projector cases as

$$
\begin{array}{ll}
\left(P_{C}^{3} M_{W}\right) u_{C}=P_{C}^{1} M_{W}, & \left(P_{C}^{3} M_{W}\right) v_{C}=P_{C}^{2} M_{W}, \\
\left(P_{P}^{3} M_{W}\right) u_{P}=P_{P}^{1} M_{W}, & \left(P_{P}^{3} M_{W}\right) v_{P}=P_{P}^{2} M_{W} .
\end{array}
$$

This can be written into a matrix equation of the form

$$
A M_{W}=0,
$$

with the design matrix

$$
A=\left(\begin{array}{c}
u_{C} P_{C}^{3}-P_{C}^{1} \\
v_{C} P_{C}^{3}-P_{C}^{2} \\
u_{P} P_{P}^{3}-P_{P}^{1} \\
v_{P} P_{P}^{3}-P_{P}^{2}
\end{array}\right) .
$$

From Eq. (9) the 3D point $M_{W}$ can be recovered from $m_{C}, m_{P}, P_{C}^{-}$, and $P_{P}$ as the null vector of the design matrix $A$, which can be obtained by a singular value decomposition (SVD) [14].

Observe that if any of the internal or external camera-projector calibration parameters change, the corresponding projection matrix is modified as $P_{i} \rightarrow P_{i}^{\prime}$, and then it is necessary to recalibrate the system.

The proposed multiresolution approach is based on a novel self-calibration method that, from a previously calibrated $3 \mathrm{D}$ rig, permits us to recalibrate it when the camera or the projector is modified, keeping the other optical system-projector or camera-fixed. The proposed self-calibration approach does not need to keep fixed the distortion and internal parameters as in [11], and additionally, this method does not need to use cues like geometric and focus or to impose a plane-movement restriction as in $[12,13]$. Two different stages are possible. In Case 1 the camera is fixed, and the projector internal and/or external calibration parameters are modified. In Case 2 the camera is modified, and the projector is fixed. The case in which both camera and projector change is not allowed.

\section{B. Case 1: Recalibration When the Projector is Modified}

In this case the camera is fixed, and the projector internal and/or external calibration parameters are modified. A scheme of the process is shown in Fig. 1. From a previously calibrated configuration, $\mathbf{C}-\mathbf{P}$ in Fig. 1, the projector is modified to a new configuration $\overline{\mathbf{P}}^{\prime}$. If the object to measure does not move with respect to the camera, we can recalibrate $\mathbf{P}^{\prime}$ using a previous 3D measure from $\mathbf{C}-\mathbf{P}$. A 3D point $M_{C}=\left[X_{C}, Y_{C}, Z_{C}, 1\right]^{T}$, referred in the camera frame (reference system positioned in the optical camera center with its $Z$ axis parallel to the optics axis), can be obtained by triangulating points $m_{P}$ and $m_{C}$. If we change the projector, these $3 \mathrm{D}$ point coordinates still are valid because there has not been any change in either the camera or the object. In the new C-P' $\mathbf{P}^{\prime}$ configuration, we compute for each camera pixel $m_{C}$ a new absolute phase measures $\left[\Phi_{x}^{\prime}\left(m_{C}\right), \Phi_{y}^{\prime}\left(m_{C}\right)\right]$ from the GC+PS technique. The projector coordinates $m_{P}^{\prime}\left(m_{C}\right)$ corresponding to $m_{C}$ are obtained from Eq. (7) as $m_{P}^{\prime}\left(m_{C}\right)=\left(\frac{\Phi_{X}^{\prime}\left(m_{C}\right) T_{X}}{2 \pi}, \frac{\Phi_{Y}^{\prime}\left(m_{C}\right) T_{Y}}{2 \pi}, 1\right)$. It is important to note that, in this case, the camera coordinates $m_{C}=\left[u_{C}, v_{C}, 1\right]$ are pixels coordinates and therefore integer values, but the corresponding projector coordinates $m_{P}^{\prime}\left(m_{C}\right)=\left[u_{P}^{\prime}\left(m_{C}\right), v_{P}^{\prime}\left(m_{C}\right), 1\right]$ have been computed from Eq. (7) at each camera pixel and are, in general, noninteger coordinates. For the corresponding points $\left[m_{C}, m_{P}^{\prime}\right]$, we have

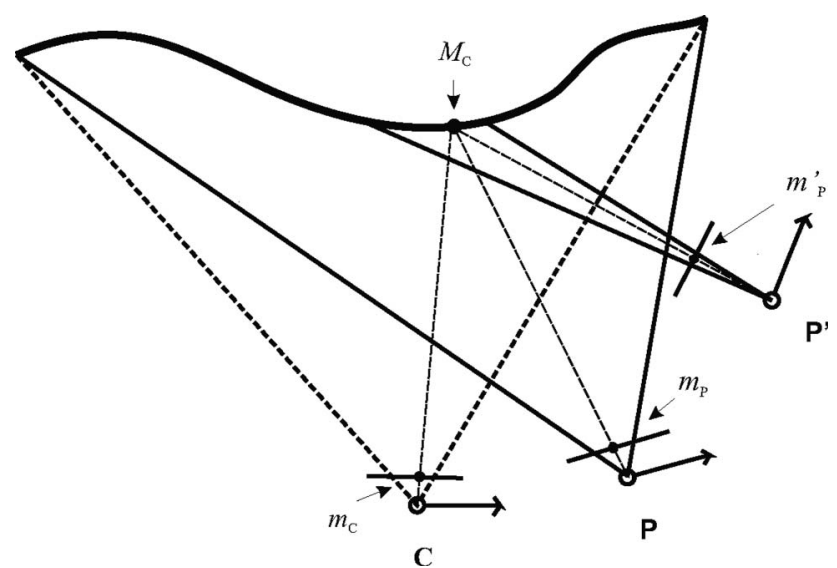

Fig. 1. Scheme of the process shown in Subsection 2.B, where $\mathbf{C}$ is the camera, $\mathbf{P}$ is the projector, and $\mathbf{P}^{\prime}$ is the projector before changing its internal and external parameters. $M_{C}$ is an arbitrary point, and $m_{P}, m_{C}$, and $m_{P}^{\prime}$ correspond to its projection in the projector $(\mathbf{P})$, camera $(\mathbf{C})$, and projector $\left(\mathbf{P}^{\prime}\right)$ frames. 
$M_{C}\left(m_{C}\right)=M_{C}\left(m_{P}^{\prime}\right)=M_{C}$. Using the correspondences $\left[m_{C}, m_{P}^{\prime}\right]$ and the $3 \mathrm{D}$ measures $M_{C}$, it is possible recalibrate the new projector configuration as shown here.

Equations (3) and (4) are rewritten in the case that we use the camera reference system instead of the world reference system as,

$$
\begin{aligned}
& s m_{C}=K_{C}[I \mid \mathbf{0}] M_{C}\left(m_{C}\right), \\
& s m_{P}=K_{P}[R \mid \mathbf{t}] M_{C}\left(m_{C}\right),
\end{aligned}
$$

where $I$ and $\mathbf{0}$ are the identity $3 \times 3$ matrix and the null $3 \times 1$ vector, respectively. On the other hand, $R$ and $\mathbf{t}$ are the rotation matrix and the translation vector that relates the camera and projector reference systems. If the projector is modified and the object to measure does not change,

$$
s m_{P}^{\prime}=P_{P}^{\prime} M_{C}\left(m_{C}\right)=K_{P}^{\prime}\left[R^{\prime} \mid \mathbf{t}^{\prime}\right] M_{C}\left(m_{C}\right) .
$$

Solving the scale factor as in Eq. ()ㅜ), we obtain

$$
\left(\begin{array}{c}
u_{P}^{\prime} P_{P}^{\prime 3}-P_{P}^{\prime 1} \\
v_{P}^{\prime} P_{P}^{\prime 3}-P_{P}^{\prime 2}
\end{array}\right) M_{C}=\left(\begin{array}{l}
0 \\
0 \\
0 \\
0
\end{array}\right)
$$

Expanding Eq. (1ㅜ) we get

$$
\left(\begin{array}{cccccccccccc}
u_{P}^{\prime} X_{C} & -X_{C} & u_{P}^{\prime} Y_{C} & -Y_{C} & u_{P}^{\prime} Z_{C} & -Z_{C} & u_{P}^{\prime} & -1 & 0 & 0 & 0 & 0 \\
v_{P}^{\prime} X_{C} & 0 & v_{P}^{\prime} Y_{C} & 0 & v_{P}^{\prime} Z_{C} & 0 & v_{P}^{\prime} & 0 & -X_{C} & -Y_{C} & -Z_{C} & -1
\end{array}\right) S_{P}=\left(\begin{array}{l}
0 \\
0
\end{array}\right),
$$

\section{Case 2: Recalibration When the Camera Is Modified}

In this case the projector is fixed, and the camera internal and/or external calibration parameters are modified. A scheme of the process is shown in Fig. 2. From a previous calibrated configuration, C-P in Fig. 2, the camera is modified to a new configuration $\mathbf{C}^{\prime}$.

If the object to measure does not move with respect to the projector, we can recalibrate $\mathbf{C}^{\prime}$ using a previous $3 \mathrm{D}$ measure from $\mathbf{C}-\mathbf{P}$. The corresponding $3 \mathrm{D}$ object point of $m_{C}$ and $m_{P}, M_{C}\left(m_{C}\right)$, can be referred in the projector frame as $M_{P}\left(m_{C}\right)=[R \mid t] M_{C}\left(m_{C}\right)$. As the projector does not image the object, the selfcalibration process here is not as straightforward as in Subsection 2.B. In the new $\mathbf{C}^{\prime}-\mathbf{P}$ configuration, we compute for each camera pixel $m_{C}^{\prime}$ a new absolute phase measures $\left[\Phi_{x}^{\prime}\left(m_{C}^{\prime}\right), \Phi_{y}^{\prime}\left(m_{C}^{\prime}\right)\right]$ from the GC + PS technique. Here the new measured absolute phases are denoted by $\left[\Phi_{x}^{\prime}, \Phi_{y}^{\prime}\right]$ to indicate that they are a new phase measurement. The corresponding projector coordinates of $m_{C}^{\prime}$ and $m_{P}^{\prime}\left(m_{C}^{\prime}\right)$ are obtained from Eq. (7) for each camera pixel as $m_{P}^{\prime}\left(m_{C}^{\prime}\right)=$ $\left(\frac{\Phi_{X}^{\prime}\left(m_{C}^{\prime}\right) T_{X}}{2 \pi}, \frac{\Phi_{Y}^{\prime}\left(m_{C}^{\prime}\right) T_{Y}}{2 \pi}, 1\right)$. Here, as in Subsection $\underline{2 . \mathrm{B}}$, $m_{C}^{\prime}=\left[u_{C}^{\prime}, v_{C}^{\prime}, 1\right]$ are pixels coordinates and therefore integer values, but the corresponding projector coordinates $m_{P}^{\prime}\left(m_{C}^{\prime}\right)=\left[u_{P}^{\prime}\left(m_{C}\right), v_{P}^{\prime}\left(m_{C}^{\prime}\right), 1\right]$ have been computed from Eq. (7) and are, in general, noninteger coordinates. Obviously we have $\left[\Phi_{x}^{\prime}\left(m_{C}^{\prime}\right), \Phi_{y}^{\prime}\left(m_{C}^{\prime}\right)\right]=\left[\Phi_{x}^{\prime}\left(m_{P}^{\prime}\right), \Phi_{y}^{\prime}\left(m_{P}^{\prime}\right)\right]$. The corresponding $3 \mathrm{D}$ point coordinate of $m_{C}^{\prime}, m_{P}^{\prime}$, and with $S_{P}=\left[P_{P, 31}^{\prime}, P_{P, 11}^{\prime}, P_{P, 32}^{\prime}, P_{P, 12}^{\prime}, P_{P, 33}^{\prime}, P_{P, 13}^{\prime}, P_{P, 34}^{\prime}\right.$, $\left.P_{P, 14}^{\prime}, P_{P, 21}^{\prime}, P_{P, 22}^{\prime}, P_{P, 23}^{\prime}, P_{P, 24}^{\prime}\right]$, where $P_{P, i j}^{\prime}$ corresponds to the $i$ th row and $j$ th column of the projector matrix in the new configuration.

Each 3D point in Eq. (14) gives us two equations. We can stack all equations to obtain an overdetermined system with typically $2 \times 640 \times 480$ rows and 12 columns that can be solved from a SVD decomposition. Once $P_{P}^{\prime}$ is computed, we can obtain from it the projector internal and the 3D rig external parameters $\left(K_{P}^{\prime}, R_{P}^{\prime}, \mathbf{t}_{P}^{\prime}\right)$ with a $R Q$ decomposition [14]. Using this initial linear estimation of $K_{P}^{\prime}, R_{P}^{\prime}, \mathbf{t}_{P}^{\prime}$, we perform a bundle adjustment nonlinear optimization to refine them and to compute the new radial and tangential distortion parameters. The radial and tangential distortion parameters are initially estimated as zero in the nonlinear refinement. In this bundle adjustment process, the retroprojected error is minimized through an iterative process [14].

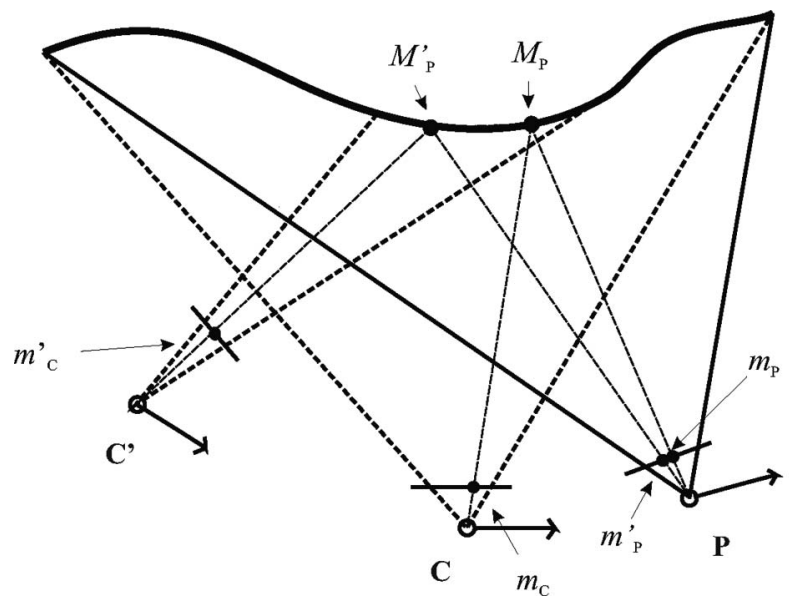

Fig. 2. Scheme of the process shown in Subsection 2.C, where $\mathbf{C}$ is the camera, $\mathbf{P}$ is the projector, and $\mathbf{C}^{\prime}$ is the camera before changing its internal and external parameters. $M_{P}$ is an arbitrary point that it is "observed" by $m_{C}$ and $m_{P} . M_{P}^{\prime}$ is another arbitrary point that it is "observed" by $m_{C}^{\prime}$ and $m_{P}^{\prime}$. 
$M_{P}^{\prime}\left(m_{C}^{\prime}\right)=M_{P}^{\prime}\left(m_{P}^{\prime}\right)=M_{P}^{\prime}$ is unknown, but as the projector and the object do not change, we can use the absolute phases as marks to obtain $M_{P}^{\prime}\left(m_{C}^{\prime}\right)$ from $M_{P}\left(m_{C}\right)$. To compute $M_{P}^{\prime}\left(m_{C}^{\prime}\right)$, it is possible to interpolate the values of $\left[m_{P}\left(m_{C}\right), M_{P}\left(m_{C}\right)\right]$ at the new points $m_{P}^{\prime}\left(m_{C}^{\prime}\right)$. Observe that this is equivalent to speaking about absolute phase or projector coordinates, because they are directly related by linear Eq. (7). The interpolation is performed at every component of $M_{P}\left(X_{P}, Y_{P}\right.$, and $\left.Z_{P}\right)$. Once we have $m_{C}^{\prime}$, $m_{P}^{\prime}\left(m_{C}^{\prime}\right)$, and $M_{P}^{\prime}\left(m_{C}^{\prime}\right)=M_{P}^{\prime}\left(m_{P}^{\prime}\right)=M_{P}^{\prime}$, it is possible to recalibrate the camera as shown here.

Equations (3) and (4) are rewritten in the case that we use the projector reference system as

$$
\begin{aligned}
& s m_{C}=K_{C}[R \mid \mathbf{t}]^{-1} M_{P}\left(m_{C}\right), \\
& s m_{P}=K_{P}[I \mid \mathbf{0}] M_{P}\left(m_{C}\right),
\end{aligned}
$$

where $R$ and $\mathbf{t}$ are again, as in Eq. (11), the rotation matrix and the translation vector that relates the camera and projector reference systems. If the camera is modified and the object to measure does not change,

$$
s m_{C}^{\prime}=K_{C}^{\prime}\left[R^{\prime} \mid \mathbf{t}^{\prime}\right]^{-1} M_{P}^{\prime}\left(m_{C}^{\prime}\right) .
$$

As mentioned in Eq. (16), we can obtain $M_{P}^{\prime}\left(m_{C}^{\prime}\right)$ from $M_{P}\left(m_{C}\right)$ by interpolation. Solving the scale factor as in Eqs. ( $\underline{8})$ and (13), we obtain

$$
\left(\begin{array}{c}
u_{C}^{\prime} P_{C}^{\prime 3}-P_{C}^{\prime 1} \\
v_{C}^{\prime} P_{C}^{\prime 3}-P_{C}^{\prime 2}
\end{array}\right) M_{P}^{\prime}\left(m_{C}^{\prime}\right)=\left(\begin{array}{l}
0 \\
0 \\
0 \\
0
\end{array}\right) .
$$

Expanding Eq. (13) we get adjustment process, the retroprojected error is minimized through an iterative process [14].

\section{Multiresolution Method}

The proposed multiresolution method is based on continuously changing the $3 \mathrm{D}$ measuring system in order to adapt its depth sensitivity to the detail size we want to inspect. If we want to increase the depth resolution of a fringe projection system, we have to change the setup, reducing the observed and projected areas and increasing the triangulation angle $[5,6,9]$.

Our proposed multiresolution approach is based on obtaining a 3D measure of the whole surface with the system previously calibrated. Next the camera or projector is changed, modifying its internal and/or external parameters. If the camera is changed, then the projector is fixed and vice versa. During the multiresolution process, the object to measure has to be unmodified. Using the self-calibrating processes explained in Subsections 2.B and 2.C, the camera or the projector are recalibrated. This process can be repeated as many times as necessary in order to obtain the desired depth sensitivity.

\section{E. Degenerate Configurations}

We have shown in Subsections 2.B and 2.C that each measured point gives us two equations to compute the projector or camera projection matrix. A general projection matrix $(P)$ has 10 degrees of freedom $\left(f^{X}\right.$, $f^{Y}, C^{X}, C^{Y}$, three rotation angles, and three components of the translation vector), therefore we need at least five points two compute it.

The problem of computing the camera or projector projection matrix, given a set of $3 \mathrm{D}$ points and its corresponding image points in the retinal plane, is known in the literature as camera resectioning [14]. As explained in [14], there are different critical

$$
\left(\begin{array}{ccccccccccccc}
u_{C}^{\prime} X_{P}^{\prime} & -X_{P}^{\prime} & u_{C}^{\prime} Y_{P}^{\prime} & -Y_{P}^{\prime} & u_{C}^{\prime} Z_{P}^{\prime} & -Z_{P}^{\prime} & u_{C}^{\prime} & -1 & 0 & 0 & 0 & 0 \\
v_{C}^{\prime} X_{P}^{\prime} & 0 & v_{C}^{\prime} Y_{P}^{\prime} & 0 & v_{C}^{\prime} Z_{P}^{\prime} & 0 & v_{C}^{\prime} & 0 & -X_{P}^{\prime} & -Y_{P}^{\prime} & -Z_{P}^{\prime} & -1
\end{array}\right) S_{C}=\left(\begin{array}{c}
0 \\
0
\end{array}\right)
$$

with $S_{C}=\left\lfloor P_{C, 31}^{\prime}, P_{C, 11}^{\prime}, P_{C, 32}^{\prime}, P_{C, 12}^{\prime}, P_{C, 33}^{\prime}, P_{C, 13}^{\prime}, P_{C, 34}^{\prime}\right.$, $\left.P_{C, 14}^{\prime}, P_{C, 21}^{\prime}, P_{C, 22}^{\prime}, P_{C, 23}^{\prime}, P_{C, 24}^{\prime}\right\rfloor$, where again $P_{C, i j}^{\prime}$ corresponds to the $i$ th row and $j$ th column of the camera matrix in the new configuration. From Eq. (18) $S_{C}$ can be computed by SVD decomposition as in Case 1. Once $P_{C}^{\prime}$ is computed, we obtain from it $K_{C}^{\prime}, R_{C}^{\prime}$, and $\mathrm{t}_{C}^{\prime}$ by an $R Q$ decomposition [14]. Finally, using the initial linear estimation of $K_{C}^{\prime}, R_{C}^{\prime}$, and $\mathrm{t}_{C}^{\prime}$, we perform a bundle adjustment nonlinear optimization to refine them and to compute the radial and tangential distortion parameters. The radial and tangential distortion parameters are initially estimated as zero in the nonlinear refinement. In this bundle configurations for camera resectioning from a single view. Most of the critical configurations shown in [14] are improbable to be found in a real calibration process from a single view except the plane configuration. If all the $3 \mathrm{D}$ points are coplanar, then there is not a unique projection matrix to model the imaging process of these $3 \mathrm{D}$ points. Therefore it is not possible to use our self-calibration method if all the $3 \mathrm{D}$ points are coplanar.

\section{Experimental Results}

The triangulation system was formed by a FireWire $1024 \times 768$ motorized zoomed camera and a modified Benq PE7700 projector, where the projection optics were modified in order to obtain a field of view from 
$1 \mathrm{~m}$ to $10 \mathrm{~cm}$. The $3 \mathrm{D}$ measuring system was calibrated using a model-based method [15].

To prove the effectiveness of the proposed technique, we tested it first with a simulation and finally with a mechanized metallic step pyramid. The simulation was performed to test the explained self-calibration method and was computed with the data used by Heikkila [16]. These data come from a real camera and are composed by $4913 \mathrm{D}$ points and their corresponding camera coordinates in the retinal plane. Using Eq. (14) or Eq. (18), we can compute $S_{P}$ or $S_{C}$ and then the corresponding internal and external parameters. Finally, by means of the bundle adjustment nonlinear optimization, we can obtain the refined calibration parameters. Figure 3 shows the Heikkila's 3D points we used to test our self-calibration process. Observe that, in Fig. 3, there are $4913 \mathrm{D}$ points distributed in two planes with a $90^{\circ}$ angle between them. The distance between nearest $3 \mathrm{D}$ points is $15 \mathrm{~mm}$.

Table 1 shows the camera calibration parameters obtained from a typical Zhang calibration and using our self-calibration method. Observe that the rotation matrices are given in the usual Rodrigues notation [17] and that the results are the same. Table $\underline{2}$ shows the retroprojected errors using the Zhang calibration method and our proposed self-calibration method. Observe that, in Table 2, the retroprojected errors in the $X$ and $Y$ camera axes for both methods are shown. As can be seen from Table 2, the computed retroprojection errors are similar in both cases and in both axes. These results show a very good determination of the calibration parameters.

The next example consists of testing the proposed multiresolution method using a calibrated mechanized step pyramid. The step pyramid has lateral dimensions of $110 \mathrm{~mm} \times 110 \mathrm{~mm}$ with four different height steps of $10,5,1$, and $0.1 \mathrm{~mm}$. Figure 4 shows an image of the pyramid with the different steps values. To prove the validity of the proposed multiresolution method, we obtained three measures in order to acquire a multiresolution measure. First we computed a $3 \mathrm{D}$ measure using a previously

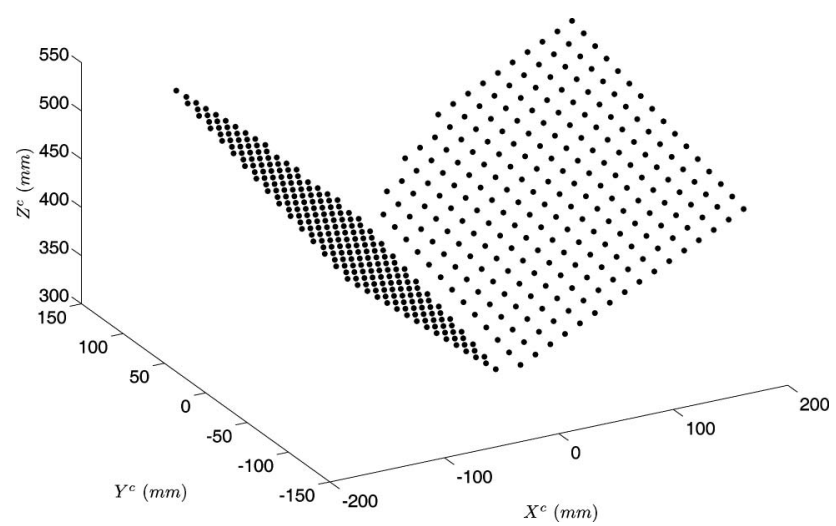

Fig. 3. Heikkila's 3D data set points, referred to in the camera reference system, used in the self-calibration process simulation. There are $4913 \mathrm{D}$ points distributed in two planes with a $90^{\circ}$ angle between them.
Table 1. Camera Calibration Parameters Obtained Using the Zhang Calibration Approach and Our Self-Calibration Method ${ }^{a}$

\begin{tabular}{lcc}
\hline & $\begin{array}{c}\text { Camera Parameters } \\
\text { (Zhang) }\end{array}$ & $\begin{array}{c}\text { Camera Parameters } \\
\text { (Self-Calibration) }\end{array}$ \\
\hline$f_{X}(\mathrm{px})$ & 1037 & 1037 \\
$f_{Y}(\mathrm{px})$ & 1034 & 1034 \\
$C_{X}(\mathrm{px})$ & 367 & 367 \\
$C_{Y}(\mathrm{px})$ & 306 & 306 \\
$k_{1}$ & -0.2 & -0.2 \\
$k_{2}$ & 0.2 & 0.2 \\
$p_{1}$ & $7 \times 10^{-5}$ & $7 \times 10^{-5}$ \\
$p_{2}$ & $-3 \times 10^{-4}$ & $-3 \times 10^{-4}$ \\
$R$ & {$[7,5,-4] \times 10^{-3}$} & {$[7,5,-4] \times 10^{-3}$} \\
$T(\mathrm{~mm})$ & {$\left[-9.98 \times 10^{1}, 1.12,5 \times 10^{-2}\right]$} & {$\left[-9.98 \times 10^{1}, 1.12,5 \times 10^{-2}\right]$} \\
\hline
\end{tabular}

${ }^{a}$ The radial and tangential distortion parameters are given by $k_{1}, k_{2}$ and $p_{1}, p_{2}$ coefficients.

calibrated configuration denoted by $\mathbf{C}-\mathbf{P}$. Second the projector was modified, reducing the projecting area and augmenting the triangulation area $\left(\mathbf{C}-\mathbf{P}^{\prime}\right)$. Finally the camera was changed, reducing the observing area and increasing the triangulation area $\left(\mathbf{C}^{\prime}-\mathbf{P}^{\prime}\right)$. Figure 5 shows a profile along the row $180 \mathrm{px}$-indicated in Fig. 4 with a black broken lineof the depth coordinate obtained with the $\mathbf{C}-\mathbf{P}, \mathbf{C}-\mathbf{P}^{\prime}$, and $\mathbf{C}^{\prime}-\mathbf{P}^{\prime}$ configurations. From Fig. 5 we can observe the appropriate registering between views, and the different depth resolutions computed in each measure. While with the $\mathbf{C}-\mathbf{P}$ configuration, we obtained a low depth resolution measure with large field of view, with the $\mathbf{C}^{\prime}-\mathbf{P}^{\prime}$ configuration, we computed a high depth resolution measure with small field of view. $\mathbf{C}-\mathbf{P}^{\prime}$ is an intermediate configuration between them. The 1,5 , and $10 \mathrm{~mm}$ steps are represented by $b$, $c$, and $d$ in Fig. 5. The smallest $0.1 \mathrm{~mm}$ step is not appreciable in Fig. 5. The step values of $b, c$, and $d$ are given in Table $\overline{3}$, where the agreement between the step heights in all configurations can be seen, and the recovered depth steps are more accurate in the fine than in the coarse measure. To show the increase of depth resolution, Fig. 6 shows a detailed profile along the row 180 px of Fig. 4 , plotting only the central region of the step pyramid. Observe that, on one hand, in Fig. 6 it is possible to see the smallest $0.1 \mathrm{~mm}$ step with $\mathbf{C}^{\prime}-\mathbf{P}^{\prime}$ configuration but not with the other cases. To compute the difference of depth recovery error in both configurations, we obtained the root mean square (rms) error in the plane central region of the step pyramid between the best fitted plane and the depth data in every configuration. The results are shown in Table 4, where a reduction factor of seven in the depth uncertainty between the $\mathbf{C}-\mathbf{P}$ and $\mathbf{C}^{\prime}-\mathbf{P}^{\prime}$ configurations can be seen.

Table 2. Retroprojected Errors Using the Zhang Calibration Method and Our Self-Calibration Engine

\begin{tabular}{lcc}
\hline & $\begin{array}{c}\text { Camera } \\
(X \text { axis })\end{array}$ & $\begin{array}{c}\text { Camera } \\
(Y \text { axis })\end{array}$ \\
\hline Retroprojection ErrorZhang $(\mathrm{px})$ & 0.06 & 0.04 \\
Retroprojection Error Self-Calibration $(\mathrm{px})$ & 0.04 & 0.03 \\
\hline
\end{tabular}




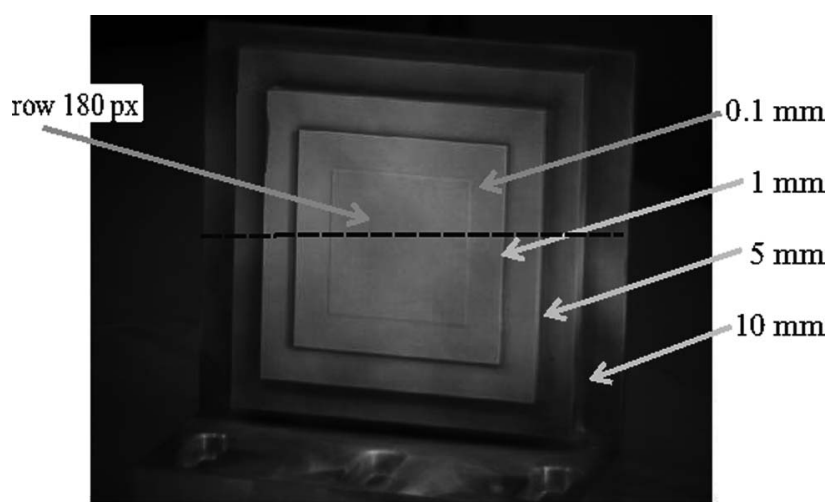

Fig. 4. Metallic step pyramid used to test the purposed multiresolution technique. The different step values are indicated. The broken dark line shows the 180 pixel row.

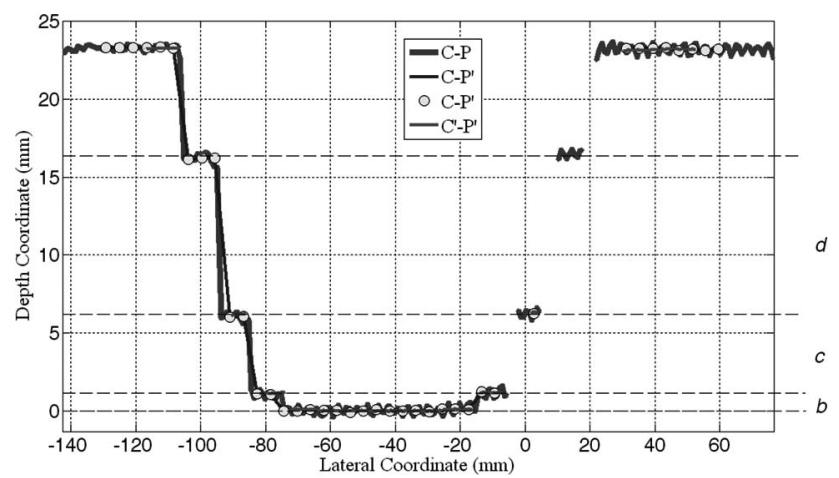

Fig. 5. Depth profile along the $180 \mathrm{px}$ row of the step pyramid obtained with the $\mathbf{C}-\mathbf{P}, \mathbf{C}-\mathbf{P}^{\prime}$, and $\mathbf{C}^{\prime}-\mathbf{P}^{\prime}$ configurations.

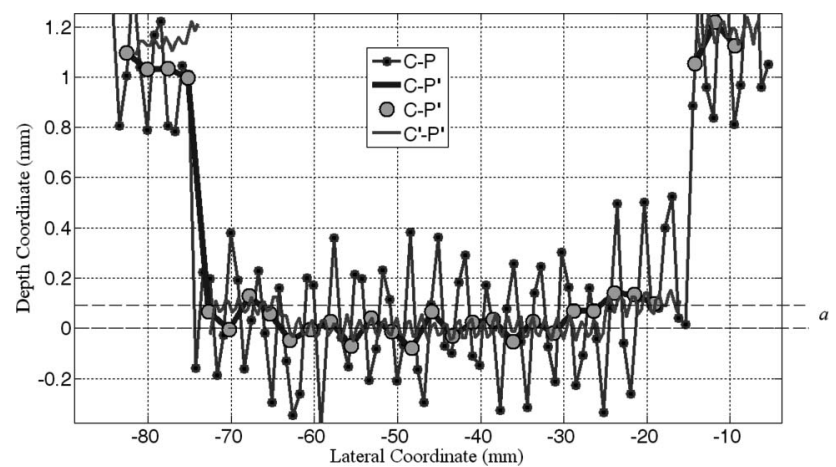

Fig. 6. Depth profile of the metallic pyramid central region to observe the $0.1 \mathrm{~mm}$ smallest step obtained with the $\mathbf{C}-\mathbf{P}, \mathbf{C}-\mathbf{P}^{\prime}$, and $\mathbf{C}^{\prime}-\mathbf{P}^{\prime}$ configurations.

Table 3. Values of the Different Depth Steps $(b, c$, and $d$ in Fig. 5) Obtained from the $C-P, C-P^{\prime}$, and $C^{\prime}-P^{\prime}$ Configurations

\begin{tabular}{llcr}
\hline & $\mathbf{C}-\mathbf{P}$ & $\mathbf{C}-\mathbf{P}^{\prime}$ & $\mathbf{C}^{\prime}-\mathbf{P}^{\prime}$ \\
\hline$d(\mathrm{~mm})$ & 9.1 & 10.1 & 10.2 \\
$c(\mathrm{~mm})$ & 4.9 & 4.94 & 5.0 \\
$b(\mathrm{~mm})$ & 0.95 & 1.1 & 1.1 \\
\hline
\end{tabular}

Table 4. Computed Root Mean Square Error Obtained in the Plane Central Region of the Step Pyramid between the Best Fitted Plane and the Depth Data in Every Configuration

$\begin{array}{lc}\text { RMS Error }(\mathbf{C}-\mathbf{P})(\mathrm{mm}) & 0.21 \\ \text { RMS Error }\left(\mathbf{C}-\mathbf{P}^{\prime}\right)(\mathrm{mm}) & 7.1 \times 10^{-2} \\ \text { RMS Error }\left(\mathbf{C}^{\prime}-\mathbf{P}^{\prime}\right)(\mathrm{mm}) & 3.0 \times 10^{-2} \\ \text { RMS }(\mathbf{C}-\mathbf{P}) / \text { RMS }\left(\mathbf{C}^{\prime}-\mathbf{P}^{\prime}\right) & 7\end{array}$

Observe that, with respect to a conventional $3 \mathrm{D}$ measurement composed of a phase measure in one direction and a further triangulation, the proposed technique needs at least six phase measurements [two coarse $(\mathbf{C}-\mathbf{P})$, two auxiliary $\left(\mathbf{C}-\mathbf{P}^{\prime}\right)$, and two fine measures $\left.\left(\mathbf{C}^{\prime}-\mathbf{P}^{\prime}\right)\right]$, a self-calibration process, and finally, a triangulation procedure. However, this increase in complexity is justified by the multiresolution character that can solve problems not affordable by a single-step triangulation setup.

\section{Conclusions}

We proposed a multiresolution method that allows us to obtain 3D measurements of large objects with high depth resolution. The proposed method does not use any registration process and overcomes the object planarwise approximation needed in [9]. This method is based on continuously changing the $3 \mathrm{D}$ measuring system through changing the camera or the projector to adapt its depth sensitivity. This process can be repeated as many times as desired, and therefore our proposed technique is very flexible.

We thank the Ministerio de Ciencia y Tecnología of Spain for the financial support of this work given by project DPI2005-03891 and the Spanish Ministry of Education for support under a FPU grant.

\section{References}

1. J. Pages, J. Salvi, R. Garcia, and C. Matabosch, "Overview of coded light projection techniques for automatic 3D profiling," in Proceedings of the IEEE International Conference on Robotics and Automation (IEEE, 2003), Vol. 1, pp. 133-138.

2. H. Steinbichler, E. H. Nösekabel, and R. Rösch, "Optical inspection in the production line," in Fringe 2001-4th International Workshop on Automatic Processing of Fringe Patterns, W. Osten and W. Jüptner, eds. (Data Science Library, Elsevier, 2001), pp. 587-592.

3. G. Notni, "360 deg shape measurement with fringe projection-calibration and application," in Fringe 20014th International Workshop on Automatic Processing of Fringe Patterns, W. Osten and W. Jüptner, eds. (Data Science Library, Elsevier, 2001), pp. 311-323.

4. G. Wiora, "High resolution measurement of phase-shift amplitude and numeric object phase calculation," Proc. SPIE 4117, 289-299 (2000).

5. D. Kayser, T. Bothe, and W. Osten, "Scaled topometry in a multisensor approach,” Opt. Eng. 43, 2469-2477 (2004).

6. P. Andrä, E. Ivanov, and W. Osten, "Scaled topometry-an active measurement approach for wide scale 3D surface inspection," in Fringe 1997: 3rd International Workshop on 
Automatic Processing of Fringe Patterns, W. Osten and W. Jüptner, eds. (Akademie-Verlag, 1997), pp. 179-189.

7. D. Kayser, T. Bothe, and W. Osten, "Fault detection in grayvalue images of surfaces on different scales," Proc. SPIE 3744, 110-117 (1999).

8. W. Osten, P. Andrä, and D. Kayser, "Highly-resolved measurement of extended technical surfaces with scalable topometry," Tech. Mess. ATM 66, 413-428 (1999).

9. J. Vargas and J. A. Quiroga, "A novel multiresolution approach for an adaptable structured light," Opt. Eng. 47, 023601 (2008).

10. M. Fiala, "Artag, an improved marker system based on artoolkit," in National Research Council Publication 47166/ ERB-1111 (2004).

11. W. Schereiber and G. Notni, "Theory and arrangements of selfcalibrating whole-body three-dimensional measurement systems using fringe projection technique," Opt. Eng. 39, 159 169 (2000)
12. S. Y. Chen and Y. F. Li, "Self recalibration of a structured light vision system from a single view," in Proceedings of the IEEE International Conference on Robotics and Automation, Washington DC, (IEEE, 2002), pp. 2539-2544.

13. Y. F. Lie and Y. Chen, "Automatic recalibration of an active structured light vision system," IEEE Trans. Robot. Autom. 19, 259-568 (2003).

14. R. Hartley and A. Zisserman, Multiple View Geometry in Computer Vision (Cambridge University, 2004).

15. R. Legarda-Sáenz, T. Bothe, and W. Jüptner, "Accurate procedure for calibration of a structured light system," Opt. Eng. 43 , 464-471 (2004).

16. J. Heikkilä, "Geometrical camera calibration using circular control points," IEEE Trans. Pattern Anal. Mach. Intell. 22, 1066-1077 (2000).

17. O. Faugueras, Three-Dimensional Computer Vision: A Geometric Viewpoint (MIT, 1993). 